\title{
LOS OBJETIVOS DE LOS VOTANTES Y LA ACCOUNTABILITY DEL GOBIERNO EN LAS ELECCIONES GENERALES DE 1993
}

\author{
VOTERS' OBJECTIVES AND THE INCUMBENT'S ACCOUNTABILITY \\ IN 1993 SPANISH NATIONAL ELECTIONS
}

\author{
JUAN RAFAEL MORILLAS MARTÍNEZ \\ Centro de Estudios Andaluces. Sevilla
}

\author{
PALABRAS CLAVE ADICIONALES \\ ADDITIONAL KEYWORDS \\ Comportamiento electoral, Voto retrospectivo, \\ Electoral Behavior, Retrospective Voting, \\ Voto prospectivo, Herestética, Accountability.

RESUMEN. En este trabajo se argumenta que las elecciones no garantizan la accountability de los gobiernos ante los ciudadanos. Se desarrolla un argumento según el cual, cuando los votantes se identifican negativamente con el partido de la oposición, los gobiernos pueden evitar su responsabilidad ante los ciudadanos mediante el desarrollo de maniobras herestéticas. Para probar empíricamente el argumento se analiza un caso especialmente relevante: las elecciones generales de 1993. Más en concreto, se analiza el comportamiento electoral de un grupo de votantes que, aun valorando negativamente la actuación gubernamental y habiendo manifestado una intención de voto distinta al partido del gobierno, finalmente le vota. Los resultados del análisis muestran cómo ciertas maniobras herestéticas permitieron al gobierno evitar su accountability ante los ciudadanos.

\begin{abstract}
In this paper, it is argued that elections per se do not guarantee the accountability of governments to citizens. An argument is put forward according to which, when citizens display a negative identification with the challenger, governments may avoid to be accountable to citizens by means of herestitical maneuvers. In order to illustrate the argument, an especially relevant test case is analyzed: the 1993 Spanish National Elections. More specifically, the empirical analysis consists of the study of the electoral behavior of a group of voters who, in spite of their negative evaluation of the government performance, and however their initial declared voting intention, they finally voted for the party in office. The empirical results show how the government avoided to be accountable by means of herestetical maneuvers.
\end{abstract}

E-mail: juanr.morillas@centrodeestudiosandaluces.es

Revista Internacional de Sociología (RIS)

Tercera Época, No 41, Mayo-Agosto, 2005, pp. 37-68. 
es determinante para que el comportamiento del agente consista en elegir los cursos de acción que materialicen los intereses de aquel. En el caso de la relación entre ciudadanos y políticos, la asimetría de información es clara. Los políticos en el poder disponen de información que los ciudadanos desconocen. Además, los políticos pueden manipular la opinión pública consiguiendo modificar las preferencias de los ciudadanos y escapar así a su control (Maravall, 1999)'. Pero es más, poco se sabe sobre el sentido en que los ciudadanos deciden su voto: sobre si su objetivo es castigar (recompensar) al partido en el gobierno, o si lo que pretenden es seleccionar al mejor de los candidatos. Si bien es cierto que existen razones para pensar que las consideraciones que determinan tal decisión son de naturaleza retrospectiva (véase Key, 1966; Fiorina, 1981; Ferejohn, 1986), también existen muchas otras para pensar que lo son de carácter prospectivo (véase Fearon, 1999). Si los ciudadanos votan de este modo, las elecciones no producen responsabilidad, simplemente porque los ciudadanos no contemplan como objetivo de su voto el cumplimiento de esta función ${ }^{2}$. En cualquier caso, lo más razonable es pensar que el objetivo de los ciudadanos es doble: pretenden que los partidos en el gobierno sean responsables ante ellos, pero también quieren seleccionar buenos tipos. Y cuando los votantes persiguen simultáneamente ambos objetivos, la consecución de la función de responsabilidad resulta más dificil aún (Fearon, 1999; Manin, Przeworski y Stokes, 1999).

La caracterización de las elecciones como una institución por medio de la cual los ciudadanos hacen a los políticos accountable ante ellos es, cuanto menos, arriesgada. El estudio de caso que se analiza en este trabajo es un ejemplo. En él se presenta un análisis de caso en el que un grupo de votantes que, aun valorando negativamente la actuación gubernamental y habiendo manifestado una intención de voto distinta al partido del gobierno, finalmente opta por este partido. Se estudian las elecciones generales de 1993; en concreto los determinantes del comportamiento de los que he llamado votantes reticentes al PSOE (partido gobernante en ese momento) (en adelante, votantes reticentes) ${ }^{3}$. Estos votantes manifestaron una intención de voto diferente al PSOE antes de la campaña debido, esencialmente, a su evaluación negativa de la actuación gubernamental, pero finalmente le votaron.

Este estudio de caso resulta especialmente interesante para ilustrar y analizar cómo los políticos pueden escapar al control de los ciudadanos ${ }^{4}$. En concreto,

\footnotetext{
${ }^{1}$ Para una versión en español de estos planteamientos, véase Maravall (2003).

${ }^{2}$ Nótese que esto no significa que la actuación gubernamental no influya en la decisión del votante. Si suponemos que el votante es racional, éste utilizará la información pasada para actualizar sus creencias sobre qué tipo de político/partido es.

${ }^{3}$ En la primera versión de este artículo, utilicé la denominación de "votantes cambiantes". Agradezco a un evaluador anónimo su sugerencia de sustituirla por la actual de "votantes reticentes".

${ }^{4}$ Obviamente, las conclusiones de este trabajo se referirán directamente al comportamiento de un grupo específico de votantes en unas elecciones concretas. Ahora bien, estas conclusiones ilustran
} 
R I S

REVISTA INTERNACIONAL DE SOCLOLOGIA

№ 41, MAYO-AG0ST0, 2005

JUAN RAFAEL MORILLAS MARTÍNEL

muestra cómo unos votantes que evalúan negativamente la actuación gubernamental, contradiciendo la caracterización de las elecciones como una institución mediante la cual los ciudadanos hacen a los políticos accountable ante ellos, votan al partido en el gobierno.

El perfil político de los votantes reticentes se caracterizó por dos tipos de variables: las político-ideológicas y una particular valoración retrospectiva de la actuación gubernamental. Políticamente eran próximos al PSOE. Ideológicamente, se situaban en una posición cercana a la que atribuían al PSOE. Además, eran ideológicamente lejanos al challenger (partido de la oposición, aspirante a alcanzar el gobierno; en adelante, partido aspirante), el PP, y tenían marcadas preferencias negativas hacia este partido político. Su evaluación retrospectiva de la actuación gubernamental, sin embargo, les alejaba del PSOE. Valoraban mal la actuación gubernamental, y por ello su intención de voto era distinta a este partido.

En el análisis posterior muestro cómo y a través de qué mecanismos, la existencia de un bad challenger influyó en el comportamiento electoral de los votantes reticentes. Considero dos mecanismos alternativos, recogidos en sendas hipótesis.

En la siguiente sección, planteo las dos hipótesis y explicito los mecanismos contenidos en cada una de ellas. Además, planteo unas consideraciones y un sencillo modelo sobre las consecuencias de la disonancia cognitiva. Estas consideraciones, si bien laterales y complementarias, no son innecesarias para la evaluación de los mecanismos por los que los votantes reticentes optaron finalmente por el PSOE. Guiará la interpretación posterior del efecto de ciertas variables explicativas sobre las probabilidades de cambio de voto al PSOE. La segunda sección, dedicada al análisis empírico, comienza con un modelo econométrico para determinar qué variables están asociadas con las probabilidades de cambio de voto al PSOE. A continuación, se evalúa la plausibilidad de la hipótesis nula, así como la de la hipótesis alternativa. Finalmente, se ofrecen unas breves conclusiones sobre el caso analizado y se discuten sus consecuencias más generales para la caracterización de las elecciones como una institución que permite conseguir la responsabilidad de los políticos ante los ciudadanos.

\section{HIPÓTESIS Y MECANISMOS}

Según la hipótesis nula — que ya formulo—, los votantes reticentes eligieron votar al PSOE, a pesar de haber manifestado una intención de voto distinta, debido al aumento de probabilidad de que el PP ganara las elecciones. Votaron, según esta

algunos de los factores que dificultan que las elecciones, por sí solas, garanticen que los políticos sean responsables ante los votantes.

40 
hipótesis, de un modo estratégico y en su decisión influyó únicamente la estimación de la probabilidad de que ganara este último. La evaluación negativa de la actuación gubernamental fue la causa de que su intención de voto fuera distinta a la representada por el PSOE. Pero el aumento de probabilidad de que ganara el $\mathrm{PP}$, resultado que les aportaba una alta des-utilidad, les condujo a optar finalmente por el PSOE a pesar de su negativa evaluación retrospectiva de la actuación del gobierno de este partido.

De acuerdo con la hipótesis alternativa, el comportamiento electoral de los votantes reticentes consistió en optar finalmente por el PSOE debido al éxito de la estrategia electoral desarrollada por el partido en el gobierno. Ésta se basó en: 1) cambiar la dirección del voto de estos ciudadanos: de retrospectivo a prospectivo, y 2) inducir el equilibrio mediante la manipulación de las preferencias de los votantes, consiguiendo que el equilibrio resultante consistiera en que los votantes reticentes optaran por el partido en el gobierno.

La explicitación de los mecanismos implícitos en estas hipótesis aclarará el contenido de las mismas.

\section{Voto estratégico}

Imaginemos que el conjunto de resultados posibles en unas elecciones es $R=\left\{r_{i}\right\}$ y el conjunto de opciones de voto de un votante, o un conjunto de ellos, dadas sus características políticas, está compuesto por dos opciones $V=\left\{v_{j}\right\}$.

Si la utilidad que obtienen los votantes de cada resultado no depende del voto que emiten ${ }^{5}$ y el individuo incorpora en su función de utilidad la estimación (subjetiva) de la probabilidad de que ocurra cada resultado condicionada al voto que emite: $\operatorname{Pr}\left(r_{i} \mid v\right)^{6}$, entonces las funciones de utilidad Von Neuman-Morgensten de los votantes son como sigue:

$$
E U\left(v_{j}\right)=\Sigma_{i} \operatorname{Pr}\left(r_{i} \mid v_{j}\right) * U\left(r_{i}\right)
$$

Donde $E U(v)$ es la utilidad esperada cuando vota $v_{j}, \operatorname{Pr}\left(r_{i} \mid v_{j}\right)$ es la estimación subjetiva por parte del votante de la probabilidad de que ocurra el resultado $r_{i}$ cuando elige $v_{j}$, $U(r)$ es la utilidad que se obtiene del resultado $r_{i}^{7}$.

\footnotetext{
${ }^{5}$ Es decir, $U\left(r_{i} \mid v_{j}\right)=U\left(r_{i}\right)$ para todo $i, j$, donde i identifica el conjunto de resultados, $R$, y $j$ un elemento del conjunto de opciones de voto $V(i=1,2,3)$.

${ }^{6}$ Es ciertamente problemático afirmar sin ninguna justificación que el votante tiene en cuenta la probabilidad de los resultados electorales condicionada a su voto, cuando un voto, es obvio, no determina por sí solo el resultado. Este es el mismo problema al que se ha enfrentado la teoría de la elección racional al explicar la decisión de votar. Sobre las "soluciones" dadas a la paradoja de no votar, así como varias réplicas a las mismas vid. Aldrich (1997).

${ }^{7}$ Puesto que he supuesto que $U(r i \mid v j)=U(r i)$ para todo $i$ y $j$.
} 


\section{RIS}

Si el votante tiene en cuenta los cambios posibles de la probabilidad de que ocurra cada resultado ${ }^{8}$ y los incorpora en su función de utilidad, entonces ésta queda como sigue:

$$
E U\left(v_{j}\right)_{i}=\Sigma_{i} \operatorname{Pr}\left(r_{i} \mid v_{j}\right)_{t}^{*} U\left(r_{i}\right)
$$

El voto es de carácter estratégico cuando el votante, a la luz de nueva información, anticipa un resultado que le lleva a sacrificar su primera preferencia por una segunda?.

Veamos una ilustración de qué es el voto estratégico (cuadro 1) de este abstracto planteamiento. Imaginemos que un votante tiene dos opciones de voto. De ellas, en ausencia de restricciones externas, prefiere la primera: $\mathrm{v}_{r}$. Las utilidades que le aporta cada resultado son $U\left(r_{1}\right)=1>U\left(r_{2}\right)=0.5>U\left(r_{3}\right)=0$. O sea, el resultado $r_{3}$ no le aporta ninguna utilidad. De los resultados que le aportan utilidad, el primero es preferido al segundo. Para simplificar el análisis, imaginemos que el votante estima las probabilidades de que ocurra cada resultado en dos momentos de tiempo. En $t=1$, la probabilidad de que ocurra $r_{3}$ es muy baja. En $t=2$, aumenta considerablemente. Supongamos unas distribuciones de probabilidad como las siguientes:

Cuadro 1.

Distribuciones de probabilidad de resultados electorales: una ilustración.

\begin{tabular}{lccccccc}
\hline $\mathrm{t}=1$ & & \multicolumn{5}{c}{$\mathrm{t}=2$} & \\
\hline & $\mathrm{r}_{1}$ & $\mathrm{r}_{2}$ & $\mathrm{r}_{3}$ & & $\mathrm{r}_{1}$ & $\mathrm{r}_{2}$ & $\mathrm{r}_{3}$ \\
$\mathrm{v}_{1}$ & 0,35 & 0,00 & 0,05 & $\mathrm{v}_{1}$ & 0,20 & 0,00 & 0,20 \\
$\mathrm{v}_{2}$ & 0,15 & 0,25 & 0,00 & $\mathrm{v}_{2}$ & 0,20 & 0,20 & 0,10 \\
\hline
\end{tabular}

Con estas distribuciones de probabilidad, las utilidades esperadas de cada opción en cada momento son las siguientes. En $t=1, E U\left(v_{1}\right)=0.400$ y $E U\left(v_{2}\right)=0.275$. Por tanto, en $t=1 v_{l}$, la opción que prefiere en primer lugar, maximiza su utilidad esperada. En $t=2$ el aumento de $\operatorname{Pr}\left(r_{3} \mid v_{1}\right)$ determina que esa opción de voto

\footnotetext{
${ }^{8}$ Por ejemplo, como consecuencia de la publicación de resultados de encuestas de intención de voto.

${ }^{9}$ Agradezco a un evaluador anónimo su sugerencia de describir la naturaleza del voto estratégico en estos términos.
}

42 
no maximice su utilidad esperada. Es la opción $v_{2}$ la que maximiza su utilidad esperada. En efecto, $\left[E U\left(v_{1}\right)\right]_{2}=0.250$ y $\left[E U\left(v_{2}\right)\right]_{2}=0.300$.

\section{Cambio del sentido del voto e inducción de un equilibrio favorable al partido en el gobierno}

Que los votantes cambiantes votaran retrospectiva o prospectivamente es una cuestión empírica. La cuestión teóricamente interesante es por qué al decidir su voto de un modo prospectivo votaron al PSOE. Aunque votaran de un modo prospectivo nada aseguraba que su voto consistiera en votar al partido en el gobierno. En efecto, éste afrontaba un importante problema de credibilidad. Si suponemos a los votantes racionales, la pasada actuación gubernamental sería utilizada por los mismos para actualizar sus creencias sobre qué tipo de partido era el PSOE (Alesina y Rosenthal, 1995: capítulo 2; Downs, 1957; Fearon, 1999). Que los votantes no decidan su voto de un modo retrospectivo no significa que la pasada actuación gubernamental no influya en la credibilidad de las propuestas del mismo.

¿Qué puede hacer el partido en el gobierno para que el equilibrio resultante en la competición le sea favorable? Una vez que los ciudadanos votan prospectivamente, los políticos pueden manipular a los ciudadanos. Esta manipulación puede ser de dos tipos. Los políticos pueden conseguir un cambio directo de las preferencias de los ciudadanos. Además, también pueden manipular a los ciudadanos en el sentido que afirma Riker $(1982 ; 1986)$. En este caso, las preferencias de los individuos están dadas: son exógenas a las estrategias de los partidos o de otros actores políticos. Con todo, los partidos pueden influir en las decisiones de los votantes. Mediante el desarrollo de maniobras herestéticas ${ }^{10}$ - por ejemplo, añadiendo dimensiones al espacio competitivo y/o controlando la agenda política-, los políticos pueden redefinir el marco en que tiene lugar la elección de los votantes. Así, en el caso de una maniobra herestética consistente en la inclusión de una nueva dimensión al espacio competitivo, al calcular la utilidad que les aporta cada uno de los resultados, los votantes tomarán en consideración las posiciones que adoptan los partidos ante esa nueva dimensión.

\section{¿Son todos los cambios de creencias reflejados en una encuesta consecuencia de una retórica exitosa?}

Como consecuencia de causas diversas y a través de mecanismos variados (como, por ejemplo, los implícitos en las hipótesis de este trabajo), un votante puede

\footnotetext{
${ }^{10}$ Riker (1990: 47) define la herestética como el cambio del espacio competitivo o de los condicionantes de la decisión de los votantes, de tal modo que la elección de éstos favorezca los intereses del promotor de la herestética.
} 
elegir una opción que no coincide con la que seleccionaría en ausencia de las mismas. Si la opción finalmente elegida es incongruente con determinadas creencias iniciales, en situaciones concretas ${ }^{11}$, el votante puede experimentar un estado de disonancia cognitiva. Ilustremos esta situación con un ejemplo (que es, a la vez, un adelanto del análisis empírico posterior). Recordemos las elecciones generales de 1993. Pensemos en los votantes que no tenían intención de votar al PSOE, tal y como manifestaron en la primera ola de una encuesta de panel, y que finalmente le votaron ${ }^{12}$. Estos votantes valoraban negativamente la actuación gubernamental $\mathrm{y}$, por ello, no tenían intención de votar al PSOE. Finalmente cambiaron su voto respecto a su primera intención y optaron por este partido. Este hecho pudo generar un estado de disonancia cognitiva en estos votantes debido a la contradicción de su evaluación de la actuación gubernamental y su comportamiento final.

¿Qué consecuencias puede tener la disonancia cognitiva? ¿Puede afectar a las creencias del individuo? Planteemos el argumento en términos formales ${ }^{13}$, a partir de los supuestos siguientes. 1) Las personas tienen preferencias no sólo sobre estados de cosas, sino también sobre sus creencias acerca de los mismos. Por tanto, los individuos no sólo tienen preferencias sobre situaciones o cuestiones materiales sino, además, sobre las visiones de tales situaciones materiales. Así, un individuo prefiere una visión del mundo no generadora de disonancia cognitiva. 2.) Las personas tienen algún control sobre sus creencias. Éstas no están totalmente determinadas, son susceptibles de modificación. Partiendo de estos supuestos, el cambio de determinadas creencias puede conducir a la eliminación, o al menos la reducción, de la disonancia cognitiva.

Dadas unas opciones de voto, $v_{i}, \mathrm{y}$ unas creencias, $C$, cada opción de voto aporta una utilidad y una des-utilidad. Puesto que el individuo tiene cierto control sobre sus creencias, éstas no son fijas. Represento este hecho con un subíndice. Por tanto, la utilidad neta que obtiene el individuo en el momento $t$ es:

$$
L\left(v_{i}\right)_{t}=U\left(v_{i}\right)-D\left(v_{i} \mid C_{i}\right)
$$

Donde $L(v)_{t}$ es la utilidad neta que le aporta votar $v_{i}$ en el momento $t, U\left(v_{i}\right)$ es la utilidad que le aporta cada opción, y $D\left(v_{i} \mid C_{t}\right)$ es la desutilidad debida a la disonancia cognitiva de votar $v_{i}$ dadas las creencias que mantiene en el momento $t$.

Imaginemos una situación en la que el votante obtiene una utilidad mayor eligiendo $v_{1}$, pero finalmente elige $v_{2}$. Si en el momento $t=1$ sus creencias son

\footnotetext{
${ }^{11}$ No planteo el argumento con pretensiones de generalidad. Mi propósito es razonar de un modo deductivo cómo se puede eliminar la disonancia cognitiva mediante un cambio de creencias.

12 Ver nota número 11.

${ }^{13}$ Para modelizaciones de las consecuencias de la disonancia cognitiva, así como varias ilustraciones de las mismas, véase Akerloff y Dickens (1982) y Rabin (1994).
} 
tales que si vota $v_{1}$ no experimenta disonancia cognitiva y si vota $v_{2}$ sí, entonces la utilidad neta es mayor si vota $v_{1}$ que si vota $v_{2}$. En efecto, puesto que $U\left(v_{1}\right)>$ $U\left(v_{2}\right)$ y $D\left(v_{1} \mid C_{1}\right)=0$ y $D\left(v_{2} \mid C_{2}\right)>0$, es inmediato que $L\left(v_{1}\right)>L\left(v_{2}\right)$. Por tanto, la opción que elige debido a la influencia de la dinámica competitiva, $v_{2}$, no es óptima dado que no maximiza su utilidad neta.

Ahora bien, si modifica sus creencias puede conseguir que la elección $\mathrm{v}_{2}$ sea óptima. Imaginemos que en $t=2$ modifica sus creencias, $C_{2}$, de modo tal que votar $v_{2}$ no le produzca disonancia cognitiva y votar $v_{1}$ sí: $D\left(v_{1} \mid C_{2}\right)>0$ y $D\left(v_{2} \mid C_{2}\right)=0$. Las utilidades que obtiene de cada voto son las mismas que en la situación anterior: $U\left(v_{1}\right)>U\left(v_{2}\right)$. En esta situación es inmediato que si $D\left(v_{l} \mid C_{2}\right)>\left[U\left(v_{1}\right)-U\left(v_{2}\right)\right]$, entonces la elección $v_{2}$, además de no ser disonante, es óptima, pues la utilidad neta de $v_{2}$ es mayor que la de $v_{1}: L\left(v_{1}\right)<L\left(v_{2}\right)$.

Estas consideraciones - que no parecen muy trabadas con la línea general de este trabajo- tienen importantes implicaciones prácticas para evitar interpretaciones apresuradas de los cambios de creencias y de actitudes medidos en encuestas de panel. Esta es la conclusión principal que se extrae de este comentario. Estas diferentes actitudes y creencias reflejadas en encuestas de panel (p.ej.: autoposicionamiento en la escala izquierda-derecha) no tienen por qué ser consecuencia exclusiva de un cambio autónomo de creencias/actitudes, independiente de las acciones previamente adoptadas. Y lo que es más importante, estos cambios de actitudes/creencias no tienen por qué ser causa de una acción (por ejemplo, votar a un partido determinado), sino que pueden ser una consecuencia de tal acción (por ejemplo, pueden ser la consecuencia de la racionalización del voto emitido).

\section{ELCOMPORTAMIENTOELECTORALDE LOS VOTANTES RETICENTES AL PSOE EN LAS ELECCIONES DE 1993}

Las elecciones generales de 1993 estuvieron marcadas, principalmente, por tres hechos. En primer lugar, el clima de corrupción política y de crisis económica que comenzó en la segunda mitad de 1992. En segundo, la aguda dinámica competitiva entre PSOE y PP. La intención de voto al PP aumentó considerablemente. Así, la diferencia de más de diez puntos porcentuales que separó a PSOE y PP en las elecciones de 1989 se redujo hasta el empate. En un momento dado, el PP incluso llegó a superar al PSOE en intención de voto. Finalmente, el resultado de las elecciones fue la victoria del PSOE al obtener casi un $4 \%$ más de votos.

Estos tres elementos conforman un puzle. ¿Cómo pudo ganar el PSOE las elecciones con esa ventaja cuando la intención de voto mostraba un empate y, especialmente, en una situación de crisis económica y aparición de casos de corrupción política? La solución: el comportamiento de los votantes que no tenían intención de votar al PSOE y finalmente lo hicieron (véase Arango y Díez, 1993). La mayoría de estos votantes estaban indecisos o pensaban optar por la abstención, 


\section{RIS}

votar en blanco o votar a otros partidos menores. Finalmente, votaron al PSOE.

En esta sección presento, en primer lugar, un modelo econométrico para determinar qué variables están asociadas al cambio de voto al $\mathrm{PSOE}^{14}$. Y en segundo lugar, evalúo la plausibilidad de las dos interpretaciones alternativas que presenté en la segunda sección de este trabajo. En concreto, examinaré la hipótesis nula según la cual el comportamiento de los votantes reticentes respondió a las características de un voto estratégico. Es decir, exploraré si el cambio de su voto respecto a su intención fue consecuencia exclusiva del aumento de la probabilidad de que el PP ganara las elecciones. Una vez que muestre la imposibilidad de aceptar esta hipótesis nula, discutiré la plausibilidad de la hipótesis alternativa -anticipada en la segunda sección-, a saber: que el comportamiento de estos electores respondió a que i) cambiaron el sentido de su voto: las consideraciones prospectivas sustituyeron a las de carácter retrospectivo en la formación de su decisión, y ii) al votar prospectivamente el equilibrio resultante fue favorable al PSOE. La tesis que defenderé es que la causa de ambos fenómenos - cambio del sentido del voto, y equilibrio favorable al PSOE- fue el éxito de la estrategia electoral desarrollada por el PSOE.

\section{Un modelo econométrico para explicar el cambio de voto al PSOE}

El modelo de regresión logística que presento en este epígrafe tiene como propósito estudiar qué variables están asociadas con el cambio de voto al PSOE. Constituye un paso previo al examen de los mecanismos alternativos especificados en las hipótesis, consistente en determinar las variables con las que está asociado el cambio de voto al PSOE. La variable endógena del modelo, VPS, es una variable dicotómica que adopta el valor 1 cuando el encuestado manifestó una intención de voto distinta al PSOE y, finalmente, votó a este partido. Adopta el valor 0 cuando no manifestó intención de votar al PSOE y, finalmente, coincidiendo con su intención, no le votó. Por tanto, quedan fuera del análisis los individuos que manifestaron intención de votar al PSOE en la primera ola del panel.

La selección de las variables explicativas del modelo responde a estos criterios. En primer lugar, se han incluido variables que permitan contrastar la plausibilidad de los mecanismos alternativos presentados en la sección anterior. Así, se han incluido variables que miden el efecto de la campaña electoral, los cambios en la ubicación en la escala ideológica de los partidos políticos principales (PSOE, $\mathrm{PP}$ e IU), así como variables que miden el papel de las consideraciones de tipo

\footnotetext{
${ }^{14}$ La encuesta a la que me refiero, y en la que baso el análisis empírico que presento en este trabajo, es la encuesta española de la serie CNEP (Comparative National Election Project). Está dirigida por Gunther y Montero. Es una encuesta de panel con dos olas: la primera se realizó un mes antes de las elecciones de 1993, y la segunda es posterior a las elecciones.
} 
estratégico en la decisión final de estos votantes. En segundo lugar, he incluido variables adicionales a las estrictamente necesarias para evaluar los mecanismos alternativos (objeto de este trabajo) con el fin de conseguir que el modelo esté completamente especificado y, de este modo, las estimaciones de los parámetros no se encuentren sesgadas. Finalmente, respecto a las preguntas que coinciden en las dos olas del panel, he incluido las variables de la primera ola y la diferencia entre el valor de la variable de la segunda ola y el de la primera. Además, he incluido la interacción de esta diferencia con una variable dummy que mide el efecto de la campaña electoral.

Así, las variables explicativas que he considerado son las siguientes:

1) Valoración de los lideres de los tres principales partidos (ANGUITA1, AZNARl y FELIPE1): medidas en una escala de 0 a 10 en la que el sentido ascendente representa una opinión más favorable.

Cambio de valoración de los líderes (ANGUITA21, AZNAR21 y FELIPE21) estas variables son la diferencia entre la valoración del líder en la segunda ola y la valoración expresada en la primera. Por tanto, los signos positivos denotan un aumento de valoración mientras que los negativos indican una disminución de la misma.

2) Proximidad a los tres principales partidos (PROXIU1, PROXPP1 y PROXP$S O E 1)$ : medida en una escala de cuatro valores. Ha sido recodificada de modo que el sentido creciente indique mayor proximidad y el decreciente, menor.

Cambio de proximidad a estos tres partidos políticos (PROXIU21, PROXPP21 y PROXPSOE21): diferencia entre la proximidad al partido en la segunda ola y en la primera. Los signos positivos, por tanto, indican aumentos de proximidad y los negativos disminuciones.

3) Ubicación de los tres principales partidos políticos en la escala ideológica y autoubicación ideológica (LRIU1, LRPP1, LRPSOE1 y LRSELF1): medidas en una escala de 1 a 10 , donde el valor 1 representa extrema izquierda y el valor 10 extrema derecha.

Diferencia de ubicación ideológica de los partidos políticos y de la autoubicación ideológica entre las olas segunda y primera (LRIU21, LRPP21, LRPSOE21 y LRSELF21): calculadas restando la ubicación en la segunda ola a la ubicación en la primera. Los signos positivos, por consiguiente, indican que el encuestado sitúa más a la derecha al partido (o a su posición ideológica) en la segunda ola; los signos negativos indican que los sitúa más a la izquierda; el valor cero indica que no cambia la posición que le atribuye al partido o a su posición ideológica.

4) PERFORMANCE: esta variable es la suma de tres variables ordinales que varían de 0 a 4 y que miden la evaluación de la actuación gubernamental (preguntas 25, 26 y 27 del cuestionario correspondiente a la primera ola del panel). La variable ha sido recodificada para que el sentido ascendente indique mejor valoración de la actuación gubernamental ${ }^{15}$. 
5) Variable $D E C I D E$ : es una variable categórica de cinco categorías que mide cuándo se decidió la opción final de voto (variable de la segunda ola). Los valores son: (4) el día antes de las elecciones, (3) unos días antes de las elecciones, (2) una semana o dos antes de las elecciones, (1) un mes antes de las elecciones, (0) mucho antes de las elecciones (ésta es la categoría de referencia utilizada en los modelos econométricos que se ha estimado $)^{16}$.

6) Las siguientes variables dummies:

- WHOWINPP: adopta el valor 1 si el encuestado manifestó en la primera ola que pensaba que el PP ganaría las elecciones y 0 en caso contrario.

- VOTE89PS: adopta el valor 1 si votó al PSOE en las elecciones generales de 1989 y 0 si no lo votó.

- FAMSAME: vale 1 si los miembros de la familia votaron al mismo partido y 0 en caso contrario.

- SPOUSAME ${ }^{17}$ : vale 1 si el compañero/a votó igual, 0 en caso contrario. CAMINT vale 1 si el encuestado manifestó que siguió la campaña electoral con mucho interés o con bastante interés, y 0 si la siguió "con ni mucho ni poco", "con poco o con ningún interés".

7) Finalmente, he incluido la interacción de la variable CAMINT con las siguientes variables:

7.1) Con el cambio de ubicación de los partidos políticos en la escala ideológica (LRPIU21, LRPSOE21 y LRPP21) para poder determinar la (posible) influencia de la campaña electoral sobre los cambios de ubicación ideológica de los partidos. Así, he estimado la significatividad de la asociación con VPS (cambio de voto al PSOE) de las siguientes interacciones: CAMINT*LRIU21, CAMINT ${ }^{*} L R P P 21$, CAMINT*LRPSOE21.

7.2) Interacción con la variable WHOWINPP: CAMINT*WHOWINPP

En un primer momento he estimado un modelo con todas estas variables. He aplicado diversos Tests de Wald ${ }^{18}$ a este modelo. Las conclusiones de estos tests han conducido al modelo definitivo en el que baso el análisis posterior (Modelo

\footnotetext{
${ }^{15}$ En la segunda ola del panel no hay preguntas sobre la evaluación de la actuación gubernamental. Por ello no, se puede medir el cambio de valoración de la actuación gubernamental.

${ }^{16}$ En la Tabla 1 estos valores aparecen representados entre paréntesis.

${ }^{17}$ Pudiera pensarse que la inclusión simultánea de las variables FAMSAME y SPOUSAME crea un problema de multicolinealidad en el modelo. No obstante, el test de multicolinealidad demuestra que no es así: todos los valores de tolerancia son superiores a 0,4 .

${ }^{18}$ La hipótesis nula de este test mantiene que los coeficientes de una variable o de un conjunto de variables explicativas son iguales a cero. $\mathrm{O}$ sea, que estas variables no están significativamente asociadas con la variable endógena. La aceptación de esta hipótesis lleva a estimar un modelo que no incluye las variables explicativas examinadas, el cual cumple la propiedad de que los parámetros obtenidos tras su estimación son insesgados.
} 
1). Presento, además del Modelo 1 , cuatro modelos más para mostrar que ciertas variables no están asociadas con el cambio de voto al PSOE (Modelos 2, 3, 4 y 5).

Tabla 1.

Modelo de regresión logística de la decisión de los votantes reticentes.

\begin{tabular}{lcc}
\hline Modelo 1 & B & Sig \\
\hline FELIPE21 & 0,7702 &, 0029 \\
PROXIU1 & 4,1055 &, 0062 \\
PROXPS1 & $-7,1609$ &, 0015 \\
PROXPS21 & $-6,7806$ &, 0011 \\
FAMSAME & 4,9836 &, 0052 \\
SPOUPSOE & 9,1998 &, 0008 \\
VOTE89PS & 3,5899 &, 0141 \\
DECIDE(1) & $-0,3418$ &, 8023 \\
DECIDE(2) & 4,1609 &, 0128 \\
DECIDE(3) & 0,6085 &, 9299 \\
DECIDE(4) & 3,3393 &, 4340 \\
WHOWINPP & 4,7603 &, 0400 \\
LRIU21 & $-0,7914$ &, 0276 \\
LRPP21 & 0,4567 &, 0606 \\
LRSELF1 & $-1,8157$ &, 0061 \\
LRSELF21 & $-1,6345$ &, 0104 \\
CAMINT & $-2,5113$ &, 0441 \\
CAMINT* & 3,1143 &, 1105 \\
WHOWINPP & & \\
Constante & $-2,1840$ &, 5760 \\
\hline
\end{tabular}

$\mathrm{R}^{2}=91,15 \%$

Casos 1 predichos $=98 \%$

Casos 0 predichos $=90 \%$

Los niveles de ajuste y de predicción del modelo definitivo (Modelo 1) son buenos. El coeficiente Nagalkerke- $\mathrm{R}^{2}$ es muy alto $(91,1 \%)$. Las variables independientes predicen correctamente el $98 \%$ de los casos en que la variable endógena adopta el valor 1 y el $90 \%$ de los casos en los que su valor es 0 . Los coeficientes son significativos para niveles de confianza muy altos (alternativamente, para niveles de significación muy bajos): mayores del $99 \%$ en la mayoría de los coeficientes y del $95 \%$ en el resto (salvo para LRPP21, que es significativo para un nivel de confianza del 94\%, la interacción de CAIMINT*WHOWINPP, que lo es al $89 \%$, y las categorías de DECIDE distintas de (2), que no son estadísticamente 
significativas). El modelo no tiene problemas de multicolinealidad (ver nota a pie de página 13).

¿Qué variables están asociadas con el cambio de voto al PSOE? El modelo de regresión logística (Modelo 1) permite ofrecer una respuesta. En primer lugar, las disposiciones iniciales fueron importantes. En concreto, la proximidad al PSOE y la lejanía a IU (como se observa en los signos de los parámetros). Además, la ideología tuvo una influencia significativa. El signo negativo del coeficiente muestra que la autoubicación a la izquierda aumenta la probabilidad de votar finalmente al PSOE ${ }^{19}$.

En segundo lugar, muestra la influencia de la congruencia con el voto del compañero/a y del resto de miembros de la familia, resultado consistente con la importancia de los grupos primarios de pertenencia en el comportamiento electoral (Lazarsfeld et al., 1968).

En tercer lugar, varias variables muestran que la cercanía al PSOE hizo posible que estos votantes, finalmente, cambiaran su voto respecto a su intención inicial. Ásí lo muestra la variable, PROXPSOE1. También fue importante el aumento de valoración del líder del PSOE (FELIPE21) y el aumento de proximidad al PSOE $\left(\right.$ PROXPSOE21) ${ }^{20}$. Además, haber sido antiguo votante del PSOE aumenta la probabilidad de votar al PSOE pese a no haber tenido intención de hacerlo.

En cuarto lugar, afirmar en la primera ola que el PP ganaría las elecciones (WHOWINPP= 1) aumenta la probabilidad del cambio de voto hacia el PSOE. El coeficiente de esta variable es muy alto, lo que indica su gran influencia en la opción final de votar al PSOE. La significación de esta variable muestra que las consideraciones estratégicas fueron importantes en la determinación del comportamiento electoral de estos votantes. Pensar que el PP ganaría las elecciones está asociado de un modo significativo con el cambio de voto respecto a la opción elegida inicialmente para acabar votando al PSOE. Además, la interacción de esta variable con la de interés en el seguimiento de la campaña, CAMINT*WHOWINPP, aumenta, igualmente, la probabilidad de terminar votando al $\mathrm{PSOE}^{21}$.

Los parámetros de los cambios de ubicación ideológica entre las olas segunda y primera de la posición del votante y de la que atribuye a IU y PP son estadísticamente significativos (LRIU21, LRPP21 y LRSELF21). El signo de los mismos indica que situar a IU más a la izquierda, al PP más a la derecha y ubicarse en

\footnotetext{
${ }^{19}$ Para un análisis más preciso de la influencia de la autoubicación ideológica en el cambio de voto, véase Barreiro y Sánchez-Cuenca (1998). Su análisis descompone la variable ideología en varios grupos y muestra qué grupos ideológicos particulares están asociados con cambiar de voto respecto a la intención inicial a favor del PSOE.

${ }^{20}$ Este efecto se caracterizó en términos periodísticos como el "tirón de Felipe González".

${ }^{21}$ En los epígrafes siguientes se exploran las implicaciones sustantivas de estas asociaciones.
} 
la segunda ola más a la izquierda aumentan la probabilidad de cambiar el voto al PSOE. La interpretación de este resultado es bastante incierta. Puesto que el panel tiene solamente dos olas, una anterior y otra posterior a las elecciones, no es posible determinar de un modo definitivo cuál de estas dos vías causales recoge la influencia de las variables de polarización ideológica: a) Los encuestados cambian su visión espacial-sitúan a IU más a la izquierda, al PP más a la derecha, y ellos se ubican más a la izquierda-y por ello deciden votar al PSOE, o b) en oposición a su intención inicial, deciden votar al PSOE y, tras ello, racionalizan su decisión modificando su visión de la situación en el continuo izquierda-derecha de los partidos IU y PP, así como su propia ubicación ${ }^{22}$.

Finalmente, el interés por la campaña electoral, por sí solo, tiene un efecto negativo sobre la probabilidad de cambio de voto al PSOE. Mostrar más interés en campaña disminuye la probabilidad de votar finalmente al PSOE ${ }^{23}$. Ahora bien, pensar que el PP ganaría las elecciones y tener interés por la campaña aumenta la probabilidad de votar al PSOE. O sea, aun pensando que el PP ganaría las elecciones, la intención de voto era distinta de $\mathrm{PSOE}^{24}$. Sin embargo, la influencia de la campaña hace que el hecho de pensar que va a ganar el PP esté asociado con un cambio de voto hacia el PSOE. Respecto a la relación entre las variables LRPP21 y LRIU21 y la variable que mide el efecto de la campaña, se podría pensar que estas variables están afectadas por la campaña. Según las interacciones de ambas variables en el modelo, esto no es así. Las interacciones de la variable CAMINT con LRIU21 y con LRPP21 no son significativas (ver Modelos 2 y 3 ). Por tanto, aceptando la variable CAMINT como proxy de la influencia de la campaña, la polarización de IU y PP

\footnotetext{
${ }^{22}$ Este es un caso de un fenómeno más general que afecta a la interpretación de la relación entre voto y autoposicionamiento de los votantes en escalas ideológicas, o de posición ante issues. La racionalización del voto consiste en que los votantes sitúan a los partidos que tienen intención de votar más cerca de su autoubicación. Por ello, la dirección de la causalidad no queda determinada: no se puede determinar si el voto sigue a la posición de los partidos en la escala o, al contrario, le precede. Véase por ejemplo, Markus y Converse (1979).

${ }^{23}$ Ciertamente, medir la influencia de la campaña electoral con esta variable es problemático. También lo es hacerlo con una variable que mida la exposición a la campaña. No parece que, por ejemplo, estar sentado frente al televisor durante horas sin prestar atención alguna tenga una influencia importante. También tiene enormes problemas medir el efecto de la campaña basándose en el cambio que experimentan algunas variables medidas en las dos olas del panel -Finkel- (1993); Finkel y Schrott (1995); Fernández (2001); Barreiro y Sánchez-Cuenca (1998)). Polarizar la situación de un partido en la escala ideológica puede ser consecuencia de factores distintos a la campaña. Por ejemplo, la racionalización del voto. Ahora bien, parece plausible pensar que si el votante manifiesta interés en el seguimiento de la campaña electoral, ésta puede influir más en su opción electoral que la de otro votante que manifiesta no tener ningún interés en seguir la campaña

${ }^{24}$ Recuérdese que la variable WHOWINPP proviene de a la primera ola.
} 
RIS

no afectó al cambio de voto al PSOE de un modo diferente a los votantes influidos por la campaña y a los no influidos. Por consiguiente, el aumento de polarización de estos partidos no parece ser consecuencia de la campaña electoral del PSOE.

En conclusión, las vinculaciones políticas (ideología, valoración de Felipe González) y personales (congruencia con el voto familiar y de la pareja) tienen una importancia enorme en el aumento de probabilidad de cambiar el voto al PSOE. Además, las consideraciones de carácter estratégico, como apuntan algunas variables explicativas del modelo, fueron importantes. La indecisión duró bastante: hasta una o dos semanas antes de las elecciones ${ }^{25}$. Resulta interesante que la valoración de la actuación gubernamental no influye en la decisión de votar al PSOE (Modelos 4 y 5). La valoración de la actuación gubernamental, precisamente, determinó que la intención de voto de estos electores fuera distinta al PSOE. La creencia de que el PP ganaría las elecciones, por sí misma, así como interactuando con la campaña, también aumentó la probabilidad del cambio de voto al PSOE.

Especialmente llamativa es la influencia de la polarización ideológica. Con independencia del mecanismo causal que canaliza su influencia, veamos qué nos indica el modelo estadístico. Pensemos en un individuo de izquierdas, que ocupa una posición de 3,5 en la escala ideológica. Los valores del resto de variables del modelo para este individuo coinciden con la media, salvo los del resto de dummies, que son cero. 1) Cuando este individuo no tiene interés por la campaña electoral ni piensa que el PP vaya a ganar las elecciones, si no cambia la ubicación de IU ni de PP, la probabilidad de que cambie su voto al PSOE es del 9\%. Si sitúa a IU un punto más a la izquierda y al PP un punto más a la derecha, la probabilidad aumenta hasta el $14 \%$. Si aumenta estas variables en dos unidades, la probabilidad es del $74 \%$. 2) Y cuando tiene interés por la campaña electoral y piensa que el PP ganará las elecciones, estas probabilidades son del $65 \%$ cuando no cambia la ubicación de IU ni de PP, del $97 \%$ cuando sitúa a IU una unidad más a la izquierda y a PP una más a la derecha y del $99 \%$ cuando lo hace en dos unidades ${ }^{26}$.

\footnotetext{
${ }^{25}$ Esto contradice una extendida interpretación dada a conocer en ciertos medios periodísticos, según la cual estos votantes manifestaron intención de no votar al PSOE porque se sentían avergonzados de que se conociera su intención real, que era votar a este partido.

${ }^{26}$ Una advertencia es necesaria. Esta simulación es una simple ilustración de las asociaciones estadísticas que hemos encontrado en los modelos estadísticos. Ahora bien, la existencia de tal asociación, no implica, por sí sola que exista una relación causal entre las variables.
} 
RIS

REVISTA INTERNACTONAL DE SOCTOLOGIA LOS OBJETIVOS DE LOS VOTANTES Y ...

$N^{\circ} 41$, MAY0-AG0ST0, 2005

Tabla 2.

Modelo de regresión logística de la decisión de los votantes reticentes.

\begin{tabular}{lcc}
\hline Modelo 2 & B & Sig \\
\hline FELIPE21 & 0,8186 &, 0032 \\
PROXIU1 & 4,3548 &, 0068 \\
PROXPS1 & $-7,6553$ &, 0021 \\
PROXPS21 & $-7,2812$ &, 0016 \\
FAMSAME & 5,3348 &, 0016 \\
SPOUPSOE & 9,7752 &, 0016 \\
VOTE89PS & 3,5638 &, 0202 \\
DECIDE(1) & $-0,4879$ &, 7295 \\
DECIDE(2) & 4,3119 &, 0132 \\
DECIDE(3) & 1,0604 &, 8868 \\
DECIDE(4) & 3,3592 &, 4937 \\
WHOWINPP & 4,8619 &, 0461 \\
LRIU21 & $-0,7597$ &, 0503 \\
LRPP21 & 0,4486 &, 1031 \\
LRSELF1 & $-1,9291$ &, 0072 \\
LRSELF21 & $-1,7612$ &, 0108 \\
CAMINT & $-2,8376$ &, 0428 \\
CAMINT & 3,7579 &, 0808 \\
*WHOWINPP & &, 6203 \\
CAMINT* & $-0,5457$ & \\
LRIU21 & & \\
CAMINT* & & \\
LRPP21 & & \\
Constante & & \\
\hline & & \\
\hline
\end{tabular}

$\mathrm{R}^{2}=91,3 \%$

Casos 1 predichos $=98 \%$

Casos 0 predichos $=92 \%$ 


\section{RIS}

REVISTA INTERNACIONAL DE SOCIOLOGIA

Tabla 3.

Modelo de regresión logística de la decisión de los votantes reticentes.

\begin{tabular}{lcc}
\hline Modelo 3 & B & Sign \\
\hline FELIPE21 & 0,7122 &, 0024 \\
PROXIU1 & 2,9875 &, 0016 \\
PROXPS1 & $-5,8548$ &, 0004 \\
PROXPS21 & $-5,2675$ &, 0004 \\
FAMSAME & 3,6769 &, 0035 \\
SPOUPSOE & 7,1073 &, 0001 \\
VOTE89PS & 2,1439 &, 0373 \\
DECIDE(1) & $-1,0930$ &, 3632 \\
DECIDE(2) & 2,6656 &, 0354 \\
DECIDE(3) & 0,2074 &, 9525 \\
DECIDE(4) & 1,2851 &, 6539 \\
WHOWINPP & 1,8539 &, 1901 \\
LRSELF1 & $-1,4166$ &, 0077 \\
LRSELF21 & $-1,2984$ &, 0131 \\
CAMINT & $-2,8539$ &, 0353 \\
CAMINT* & 3,9761 &, 0580 \\
WHOWINPP & & \\
CAMINT* & $-0,9673$ &, 1141 \\
LRIU21 & 0,1235 &, 7845 \\
CAMINT* & 1,3228 & \\
LRPP21 & & \\
Constante & & \\
\hline
\end{tabular}

$\mathrm{R}^{2}=89,8 \%$

Casos 1 predichos $=98 \%$

Casos 0 predichos $=90 \%$

54 
Tabla 4.

Modelo de regresión logistica de la decisión de los votantes reticentes.

\begin{tabular}{lcc}
\hline Modelo 4 & B & Sig \\
\hline FELIPE21 & 0,7206 & 0,0066 \\
PROXIU1 & 4,2202 & 0,0071 \\
PROXPS1 & 7,0999 & 0,0018 \\
PROXPS21 & 6,7427 & 0,0012 \\
FAMSAME & 5,0597 & 0,0049 \\
SPOUPSOE & 9,2921 & 0,0010 \\
VOTE89PS & 3,6445 & 0,0159 \\
DECIDE(1) & $-0,3583$ & 0,7917 \\
DECIDE(2) & 4,1009 & 0,0179 \\
DECIDE(3) & 0,7841 & 0,9057 \\
DECIDE(4) & 3,5290 & 0,4289 \\
WHOWINPP & 4,9602 & 0,0403 \\
LRIU21 & $-0,8450$ & 0,0266 \\
LRPP21 & 0,4641 & 0,0614 \\
LRSELF1 & $-1,7810$ & 0,0067 \\
LRSELF21 & $-1,6509$ & 0,0103 \\
CAMINTDU & $-2,7341$ & 0,0402 \\
CAMINT* & 3,2593 & 0,1026 \\
WHOWINPP & & 0,8321 \\
PERFORMANCE & 0,0690 & 0,5430 \\
Constante & $-3,3573$ & \\
\hline
\end{tabular}

$\mathrm{R}^{2}=91,1 \%$

Casos 1 predichos $=98 \%$

Casos 0 predichos $=90 \%$ 
Figura 1.

Probabilidad de cambiar el voto al PSOE. Efecto combinado de la campaña, creer que PP ganaria las elecciones y polarización de la posición de PP e IU.

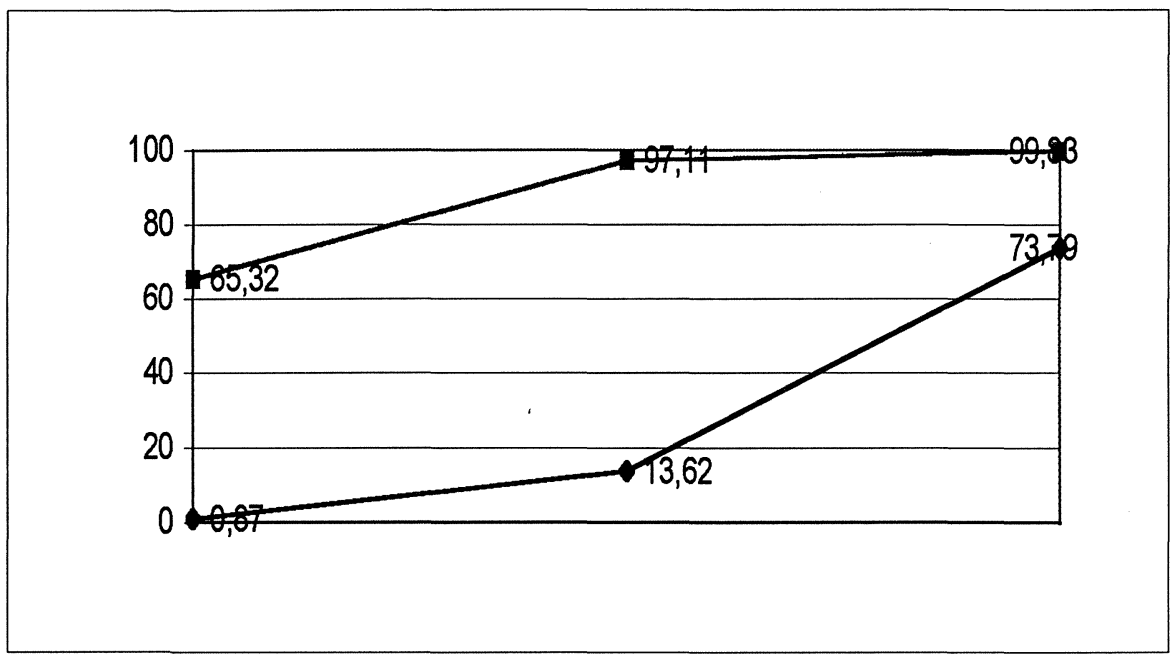

Finalmente, nótese que la variable PERFORMANCE no está asociada con el cambio de voto al PSOE (Modelo 4). Tampoco lo está la interacción de ésta con la variable CAMINT. Por tanto, la evaluación retrospectiva de la actuación gubernamental no influyó en el cambio de voto al PSOE.

Los mecanismos alternativos para explicar el comportamiento electoral de los votantes reticentes al PSOE

\section{La hipótesis nula: voto estratégico}

Recordemos la expresión (2) [véase la sección segunda de este trabajo]. La utilidad esperada de la elección del votante depende de la estimación que éste hace de la probabilidad de cada resultado condicionada al voto que emite, así como de la utilidad que obtiene de cada resultado. Los cambios de estimación de aquéllas probabilidades, dado que las utilidades que obtiene de cada resultado son fijas, pueden determinar cambios en la elección que hace máxima la utilidad esperada en un momento $t$. En efecto, el comportamiento estratégico consiste en que un cambio de la probabilidad de que ocurra un resultado no deseado hace que se elija una opción de voto que no coincide con la primera preferencia. Este comportamiento es consecuencia exclusivamente de los cambios en las probabilidades de cada resultado, y no de cambios en las utilidades asociadas a los resultados. 
Tabla 5.

Modelo de regresión logística de la decisión de los votantes reticentes.

\begin{tabular}{|c|c|c|}
\hline Modelo 5 & B & Sig \\
\hline FELIPE21 & 0,7188 &, 0083 \\
\hline PROXIU1 & 4,6867 &, 0122 \\
\hline PROXPS1 & $-7,6431$ &, 0026 \\
\hline PROXPS21 & $-7,1214$ &, 0020 \\
\hline FAMSAME & 5,0091 &, 0066 \\
\hline SPOUPSOE & 9,9404 &, 0024 \\
\hline VOTE89PS & 3,7986 &, 0184 \\
\hline $\operatorname{DECIDE}(1)$ & $-0,8942$ &, 5447 \\
\hline $\operatorname{DECIDE}(2)$ & 3,9601 &, 0247 \\
\hline $\operatorname{DECIDE}(3)$ & 0,9618 & ,9295 \\
\hline $\operatorname{DECIDE}(4)$ & 3,3091 &, 5820 \\
\hline WHOWINPP & 4,8598 &, 0571 \\
\hline LRIU21 & $-0,8416$ &, 0374 \\
\hline LRPP21 & 0,4380 &, 0856 \\
\hline LRSELF1 & $-1,9687$ &, 0083 \\
\hline LRSELF21 & $-1,9057$ &, 0142 \\
\hline CAMINT & $-6,4573$ & , 1588 \\
\hline $\begin{array}{l}\text { CAMINT* } \\
\text { WHOWINPP }\end{array}$ & 3,7470 &, 0795 \\
\hline PERFORMANCE & $-0,2684$ &, 5941 \\
\hline $\begin{array}{l}\text { CAMINT* } \\
\text { PERFORMANCE }\end{array}$ & 0,6467 &, 3852 \\
\hline Constante & $-1,4480$ &, 8076 \\
\hline
\end{tabular}

$\mathrm{R}^{2}=91,3 \%$

Casos 1 predichos $=98 \%$

Casos 0 predichos $=95 \%$ 
¿Puede caracterizarse el comportamiento electoral de los votantes reticentes como voto estratégico? ¿Votaron finalmente al PSOE, cambiando su intención inicial, debido únicamente a que aumentó su estimación de la probabilidad de que ganara el PP las elecciones?

El perfil de estos votantes se caracteriza por dos elementos: son críticos con la actuación del gobierno y son políticamente próximos al PSOE.

En las elecciones generales de 1993, el conjunto de resultados posibles que podían prever los votantes, basándose en los resultados de las encuestas de intención de voto publicadas antes de las elecciones, fue el siguiente: $R=\left\{r_{1}, r_{2}, r_{3}\right\} . r_{1}$ : gana PSOE, pero con una ventaja reducida, $r_{2}$ : PSOE gana con mayor margen y $r_{3}:$ PP gana las elecciones. Las opciones de voto de los votantes reticentes fueron básicamente dos: $V=\left\{v_{1}, v_{2}\right\} ; v_{1}$ : no votar al PSOE para mostrar su descontento con la actuación gubernamental (lo que, en términos periodísticos, se llamó voto de castigo) y $v_{2}$ : votar al PSOE, dado su perfil político.

Para contrastar empíricamente si votaron estratégicamente necesitamos conocer: 1 la utilidad que aportaba a estos votantes cada resultado, y 2 si al cambiar la estimación de la probabilidad de que el PP ganara las elecciones su opción de voto continuaba siendo $v_{1}$ (voto de castigo) ${ }^{27}$. No disponemos de datos que posibiliten calcular las funciones de utilidad de los votantes ni que nos permitan conocer las probabilidades subjetivas de realización de los resultados $r_{1}, r_{2}$ y $r_{3}$ Ahora bien, es posible inferir ambos grupos de variables a partir del perfil político de los reticentes y de la evolución de las estimaciones de intención de voto de encuestas previas a la fecha de las elecciones.

1. El perfil de los votantes reticentes al PSOE - los votantes recogidos en el valor 1 de la variable VPS - ha sido analizado por Barreiro y Sánchez-Cuenca (1998). Las conclusiones de su análisis empírico inciden en que i) valoraban negativamente la actuación gubernamental (su valoración coincide con la de los electores que no votaron al PSOE) y ii) eran políticamente cercanos al PSOE. En efecto, podemos reafirmar el segundo de estos puntos basándonos en los siguientes argumentos: 1) su ubicación en la escala ideológica es la izquierda, entre el centroizquierda y la extrema izquierda; 2) valoran positivamente a Felipe González; 3) muchos eran antiguos votantes del PSOE en 1989; 4) situaban al PP muy a la derecha - la ubicación media del PP por parte de estos votantes es 8.5 y su autoubicación es 3.7 (datos de la segunda ola), con lo cual están ideológicamente muy alejados del PP-. Además, tienen marcadas preferencias negativas hacia el PP (Marí-Klose, 1997) y, finalmente, 5) las personas de su entorno social eran votantes del PSOE.

\footnotetext{
${ }^{27}$ Ver nota número 4.
} 
Por tanto, parece plausible que las utilidades que aportan a estos votantes cada uno de estos resultados estén ordenadas del siguiente modo: $U\left(r_{1}\right)>U\left(r_{2}\right)>U\left(r_{3}\right)$. En efecto, el resultado $r_{1}$ (gana el PSOE pero por un escaso margen) es coherente con su evaluación negativa de la gestión gubernamental y con sus características políticas. El resultado $r_{2}$ es coherente con sus características políticas, pero no su evaluación (negativa) retrospectiva de la actuación gubernamental. Finalmente, el resultado $r_{3}$ es el que les aporta menor utilidad, dada su lejanía política al PP.

2. La publicación de encuestas de intención de voto fue cambiando la estimación acerca del resultado electoral final fuera uno $u$ otro ${ }^{28}$.

2.1.) Desde la fecha de las elecciones de 1989 hasta finales de 1991 la distancia en intención de voto entre PSOE y PP coincidió con la diferencia porcentual de votos obtenidos por cada partido en las elecciones de 1989 (entre un 13 y un $14 \%)$.

2.2.) Desde los primeros meses de 1992 hasta el primer trimestre de 1993 el PP redujo considerablemente su distancia respecto al PSOE. Esta reducción de la distancia en intención de voto coincidió con el desarrollo de evaluaciones muy críticas de la situación económica y política, así como de la actuación gubernamental. Los votantes del PSOE en 1989 que no tenían intención de votarle de nuevo en las elecciones de 1993 mostraron esa misma actitud crítica. Con todo, todavía en marzo de 1993 más de la mitad de los electores pensaba que el PSOE sería el ganador de las elecciones ${ }^{29}$. Por tanto, la situación en este período se caracterizó por la valoración negativa de la actuación gubernamental, la disminución de la intención de voto al PSOE y la creencia de que este partido acabaría ganando las elecciones. Por ello, parece claro que la valoración negativa de la actuación gubernamental determinó que estos votantes reticentes y/o críticos del PSOE decidieran no volver a votar al PSOE. Puesto que la estimación subjetiva de la probabilidad de que el PSOE ganara las elecciones era alta, esta decisión no tendría el coste de que el PP ganara las elecciones. Por tanto, cuando la estimación de la probabilidad de que el PP ganara las elecciones era baja, la elección de la opción que maximizaba su utilidad esperada era $v_{1}$ ("voto de castigo" al PSOE).

2.3) En el primer trimestre de 1993 la diferencia de intención de voto entre 'SOE y PP se redujo tanto que en marzo las encuestas daban un empate entre nbos partidos. Incluso el PP aventajó al PSOE en algunos momentos (ver

la 2). La posibilidad de que el PP ganara las elecciones era real. La evaluazon subjetiva de los resultados mostró a principios de mayo que solo el $38 \%$

${ }^{28}$ Véase a Arango y Díez (1993).

${ }^{29} \mathrm{El}$ dato que debería utilizar es la evaluación subjetiva de los resultados de las elecciones por parte de los votantes reticentes. Sin embargo, no dispongo de ese dato. Ahora bien, no existen razones poderosas para suponer que este grupo de votantes tuvo una percepción diferente al resto de electores. 
de los encuestados consideraba que el PSOE volvería a ganar las elecciones ${ }^{30}$. La evolución de la intención de voto refleja una ligera ventaja del PP sobre el PSOE desde marzo hasta principios de mayo, momento en que llegan a empatar. A mediados de mayo el PSOE obtiene una ligera ventaja y finalmente gana las elecciones al obtener un 3,9\% más de votos que el PP. En la tabla 6 se observa que la abstención disminuyó desde marzo. Igual ocurre con la indecisión sobre el voto a emitir. La comparación de las intenciones de voto con el voto final en la encuesta de panel DATA93 indica que la mayoría de indecisos y abstencionistas, según manifestaron antes de las elecciones, terminaron votando al PSOE.

Por tanto, parece existir una asociación entre a) aumento del porcentaje de votos del PSOE, b) reducción de la abstención y de la indecisión, y c) cambio de la valoración subjetiva de la probabilidad de que el PSOE o el PP ganaran las elecciones (en marzo un $61 \%$ de encuestados pensaba que el PSOE ganaría las elecciones; a comienzos de mayo solo un 38\%). Según la hipótesis nula, el aumento de probabilidad de que el PP ganara las elecciones si seguían optando por emitir un voto de castigo al PSOE determinó que los votantes reticentes, finalmente, optaran por votar al PSOE $\left(\mathrm{v}_{2}\right)$. Según esta interpretación, la $\operatorname{Pr}\left(r_{3}\left|v_{p_{2}}\right\rangle\right.$ $\operatorname{Pr}\left(r_{3} \mid v_{1}\right)_{1}$; e incorporando estos cambios en el cálculo de la utilidad esperada de cada opción de voto $U\left[\left(v_{1}\right)\right]_{2}<U\left[\left(v_{2}\right)\right]_{2}$. Por ello, estos votantes eligieron $v_{2}$ : votar al PSOE.

Es cierto, como muestran los datos de la Tabla 6, que existe una asociación entre intención de voto al PSOE, disminución de la indecisión y de la abstención y aumento de los votos PP. Ahora bien, ¿existe una relación de causalidad entre estas variables: entre el aumento de la intención de voto al PP según las encuestas de opinión y cambiar el voto respecto a la intención? ¿Consiste el mecanismo que conecta el aumento de probabilidad de que ganara el PP las elecciones y cambiar el voto respecto a la intención votando al PSOE simplemente en el implicado en el voto estratégico? Por tanto, ¿no podemos rechazar la hipótesis nula?

La interpretación de los parámetros del modelo econométrico presentado conduce a todo lo contrario: al rechazo de la hipótesis nula. En efecto, la variable WHOWINPP, que pertenece a la primera ola del panel, aumenta la probabilidad de cambiar de voto al PSOE sin haber tenido intención de votar a este partido. O sea, inicialmente, en la primera ola del panel - antes de las elecciones - los votantes reticentes simultáneamente 1) pensaban que el PP ganaría las elecciones, y 2) no tenían intención de votar al PSOE. Además, 3) valoraban mal la actuación gubernamental, razón por la que su intención de voto era - a pesar de su perfil político-ideológico- distinta del PSOE. Por tanto, aun pensando que el PP ganaría las elecciones, no mostraron intención de votar al PSOE. La causa de ello es la evaluación negativa de la actuación guber-

${ }^{30}$ Ver nota anterior. 
Tabla 6.

Evolución de la intención de voto en el periodo marzo-mayo de 1993.

\begin{tabular}{lccccc}
\hline & Marzo & Abril & Mayo I & Mayo II & Result,93 \\
\hline PSOE & 33,2 & 33,5 & 33,8 & 35,4 & 38,7 \\
PP & 34,5 & 34,5 & 33,8 & 34,0 & 34,8 \\
Abstención & 32,0 & 29,0 & 28,0 & 27,6 & 23,0 \\
Indecisos & 16,4 & 14,0 & 11,0 & 8,2 & - \\
\hline
\end{tabular}

Fuente: Arango y Díez (1993: 14).

namental. Si la hipótesis del voto estratégico fuera cierta, pensar que el PP ganaría las elecciones y manifestar una intención de voto diferente al PSOE serían hechos incompatibles que no podrían suceder simultáneamente. En efecto, de acuerdo con la interpretación según la cual el comportamiento de los votantes cambiantes se puede caracterizar como voto estratégico, éstos votaron al PSOE modificando su intención primera porque la posibilidad de que el PP ganara las elecciones les condujo a elegir su segunda opción de voto: votar al PSOE.

En la segunda ola, el modelo econométrico ya refleja la desaparición de la compatibilidad entre pensar que el PP ganaría las elecciones (WHOWINPP $=1$ ) y no votar al PSOE. Si bien en un primer momento el hecho de pensar que el PP ganaría las elecciones fue compatible con no tener intención de votar al PSOE, en la segunda ola pensar que el PP sería el vencedor de las elecciones aumenta la probabilidad de votar, cambiando la intención de voto al PSOE. Por tanto, entre las dos olas del panel algo ocurrió para que la creencia de que el PP ganara las elecciones hiciera a estos votantes optar por el PSOE, renunciando con ello a su intención inicial. Además, así lo muestra la significatividad y el signo positivo del parámetro de la interacción de la variable efecto de campaña y la variable que mide pensar que ganaría el PP(CAMINT*WHOWINPP). Por tanto, podemos rechazar la hipótesis nula: la interpretación del comportamiento de los votantes reticentes como voto estratégico no es plausible.

\section{Una hipótesis alternativa}

En efecto, algo tuvo que ocurrir para que el hecho de creer que el PP ganaría las elecciones dejara de ser compatible con no votar al PSOE. Como se observa en el Modelo 1 [Tabla 1], además de WHOWINPP, la interacción de esta variable con aquella que mide el interés en el seguimiento de la campaña, CAMINT, contribuye positivamente y de un modo significativo a aumentar la probabilidad de votar al PSOE pese a no haber tenido intención de hacerlo. Este efecto de la 
RIS

campaña consiste en provocar que los que pensaban que el PP ganaría las elecciones cambiaran su intención y votaran finalmente al PSOE.

Algunos análisis de las elecciones generales de 1993 - replicando la recuperación de las ideas de Lazarsfeld et al. (1968) realizada en los trabajos de Finkel (1993) y Finkel y Schrott (1995) — han concluido que el comportamiento electoral de los votantes reticentes fue una consecuencia de la campaña electoral del PSOE. Ésta, simplemente activó a estos votantes: despertó sus disposiciones políticas iniciales (recuérdese la descripción del perfil político de estos votantes, en concreto su cercanía al PSOE) y éstos votaron conforme a las mismas (vid. Fernández, 2001: 31; Barreiro y Sánchez-Cuenca, 1998). El problema de esta interpretación consiste en que no ofrece una explicación de por qué la evaluación retrospectiva de la actuación gubernamental deja de importar para explicar el voto final al PSOE y son solamente esas disposiciones políticas iniciales las determinantes. La evaluación negativa de la actuación del gobierno fue la causa de que su intención de voto inicial fuera distinta del PSOE. Sin embargo, en el momento de las elecciones, a pesar de dicha evaluación de la actuación gubernamental acaban votando al PSOE. Además, de acuerdo con el modelo de regresión logística presentado, no son solamente esas disposiciones iniciales las que importan.

Ciertamente, y de acuerdo con el modelo de regresión logística presentado, la campaña electoral influyó en la probabilidad de cambio de voto al PSOE. Pero su efecto no consistió en una simple activación, en apretar un botón y encender unas disposiciones políticas que estaban apagadas. Mediante su efecto, pensar que el PP ganaría las elecciones deja de ser compatible con no votar al PSOE. En efecto, la interacción CAMINT*WHOWINPP está asociada con un aumento significativo de la probabilidad de cambiar el voto respecto a la intención inicial y optar finalmente por el PSOE. Además, la variable PERFORMANCE (Modelo 4) deja de importar en la explicación del comportamiento electoral. En la primera ola, la mala evaluación retrospectiva de la actuación gubernamental es la causa de que aun pensando que el PP ganaría las elecciones, la intención declarada por estos votantes sea distinta al PSOE. Pero en la segunda ola, tal evaluación de la actuación gubernamental deja de ser incompatible con votar al PSOE, y pensar que el PP ganaría las elecciones pasa a ser incongruente con no votar a este partido. O sea, dos fenómenos ocurren simultáneamente - negativa evaluación de la actuación gubernamental y votar al PSOE-, y otros dos dejan de tener lugar a la vez: pensar que el PP ganaría y no votar al PSOE. En la Figura 2 se observa la influencia de las variables CAMINT y WHOWINPP en las probabilidades de terminar votando al PSOE pese a haber manifestado intención de no hacerlo. Para un individuo caracterizado por variables explicativas que adoptan en valor de la media en las variables explicativas continuas y cero en las variables dummies, este efecto es el siguiente: 1) Cuando no piensa que el PP ganará las elecciones y tampoco ha seguido con interés la campaña electoral, su probabilidad de votar finalmente al PSOE es muy baja (1\%); 2) si pensó que el PP ganaría las elecciones, 
Figura 2.

Probabilidad de cambiar el voto al PSOE. Efecto combinado de la campaña y de creer que el PP ganaria las elecciones.

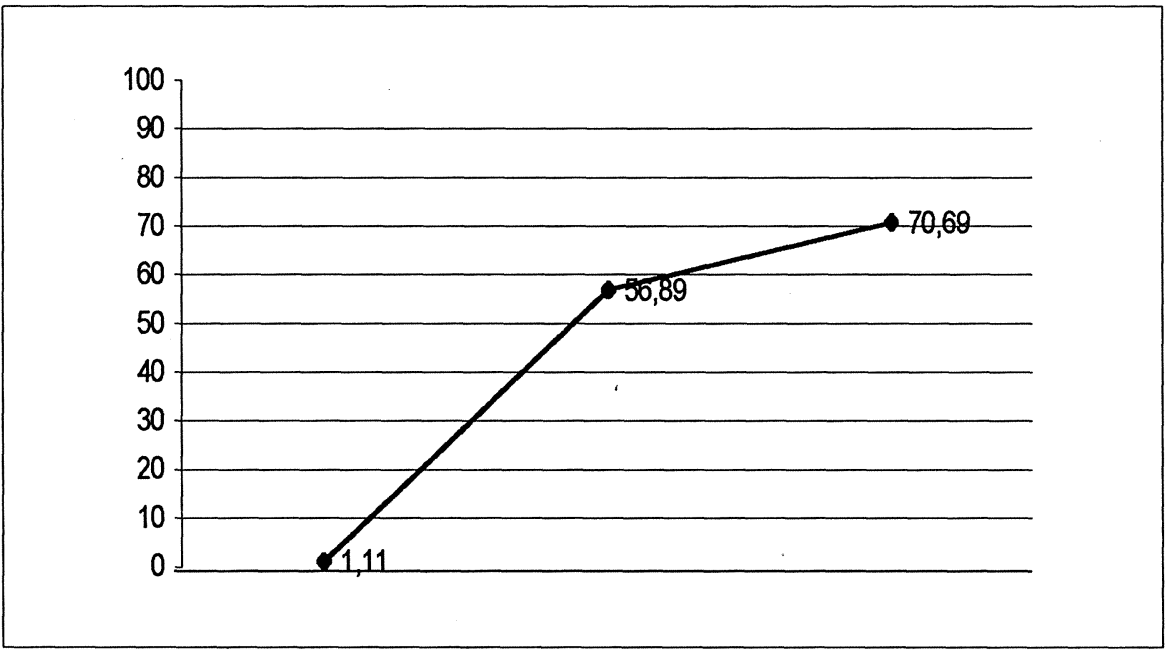

esta probabilidad aumenta hasta el $57 \%$; 3) pero si además siguió la campaña con interés, esta probabilidad alcanza el $71 \%{ }^{31}$

La interpretación de la decisión de estos votantes no es inmediata. No dispongo de las variables necesarias para dar una respuesta definitiva ${ }^{32}$ pero, aunando la información que aporta el modelo econométrico y la información cualitativa sobre la campaña electoral del $\mathrm{PSOE}^{33}$, es posible ensayar una interpretación ${ }^{34}$. Así, la

\footnotetext{
${ }^{31} \mathrm{La}$ advertencia de la nota 26 se aplica también en este caso.

${ }^{32}$ El cuestionario de DATA 93 no incluye preguntas que permitan construir variables que midan el sentido del voto - retrospectivo o prospectivo-, ni variables que midan la posición ante issues de los partidos políticos y de los votantes.

${ }^{33} \mathrm{Me}$ baso en el detallado análisis de contenido realizado por Marí-Klose (1997), basado en el seguimiento campaña realizado por el Diario El Pais. Dos elementos caracterizaron esta campaña: conseguir que los votantes tomaran su decisión de un modo prospectivo y resaltar la naturaleza y las señas de identidad del PP. El título de la campaña del PSOE, Votar Futuro, revela el primer elemento. El segundo se materializó en los mensajes transmitidos en la campaña del PSOE.

${ }^{34}$ Es cierto que, seguramente, no todos los votantes reticentes conocieran el seguimiento de la campaña que realizó el diario El Pais. Por eso, más que un análisis, el comentario que sigue a continuación en el texto principal es una "interpretación". Agradezco a un evaluador anónimo esta observación.
} 
RIS

REVISTA INTERNACIONAL DE SOCIOLOCIA

№ 41, MAYO-AGOST0, 2005

JUAN RAFAEL MORILLAS MARTÍNEL

interpretación que aquí se ofrece de las asociaciones encontradas en el Modelo 1 es la siguiente. Un primer efecto de la campaña consistió en cambiar el sentido del voto: que la formación de la decisión de los votantes tuviera lugar de un modo prospectivo y no retrospectivo. Ahora bien, aun siendo esta interpretación correcta, no es suficiente para explicar por qué votaron al PSOE; por qué el equilibrio, para estos votantes, consiste en votar PSOE. La cuestión, en efecto, no es baladí. No se puede responder, sin más, que el equilibrio implicaba votar al PSOE. Esta apresurada interpretación olvida que, aunque el sentido del voto sea prospectivo, la pasada actuación gubernamental influye en la credibilidad de las propuestas del partido en el gobierno (Alesina y Rosenthal, 1995: capítulo 2; Downs, 1957; Fearon, 1999). Queda por responder u ofrecer una interpretación acerca de por qué el equilibrio implicaba para estos votantes elegir al PSOE, no obstante los importantes problemas de credibilidad que afrontaba este partido.

Nuevamente tengo que recurrir a la combinación de las conclusiones del modelo regresión con información cualitativa sobre la campaña del PSOE. La campaña del PSOE incidió en la existencia de un programa oculto del PP. Según esta interpretación de los líderes del PSOE, una vez que el PP llegara al gobierno, si resultaba elegido, desarrollaría un programa no de centro, sino de derechas.

Además, el PSOE insistió en la naturaleza y las señas de identidad del PP: era un partido de derechas. Traduciendo estas acciones al lenguaje de las teorías que estoy utilizando, especialmente la Teoría espacial del voto, el PSOE: 1) incluyó la dimensión izquierda-derecha para marcar las identidades de los partidos - de este modo, la competición por el voto, una vez conseguido que el sentido de éste fuera prospectivo, era favorable al $\mathrm{PSOE}^{35-}$; e 2) incidió en disminuir la credibilidad del PP para compensar así la merma de su propia credibilidad debida a su (pasada) actuación gubernamental.

La influencia de la inclusión de la dimensión izquierda-derecha pudo ser efectiva gracias a dos mecanismos - discutidos en la primera sección del trabajo: retórica o herestética. ¿Cuál de los dos fue el que indujo el equilibrio hacia el PSOE? El modelo econométrico nos informa que situar al PP más a la derecha en la segunda ola del panel aumenta la probabilidad de votar al $\mathrm{PP}^{36}$. Una interpretación precipitada atribuiría este efecto a que el PSOE hizo creer a los votantes reticentes que el PP era más de derechas, lo que modificó sus preferencias y, como consecuencia, terminaron por votar al PSOE. Es decir, la retórica del PSOE

\footnotetext{
${ }^{35}$ Siempre suponiendo que los votantes reticentes resultaran influidos por este mensaje.

${ }^{36}$ Idéntico efecto tuvo el hecho de situar más a la izquierda a IU. Como el coeficiente del parámetro de LRIU21 es negativo, situar a IU más a la izquierda (LRIU21) determina aumentos de la probabilidad de votar a IU.
} 
convenció a los votantes reticentes. Ahora bien, ésta no es la única respuesta posible. Puede ocurrir que situar a PP más a la derecha no sea una causa, sino una consecuencia de cambiar el voto hacia el PSOE, esto es, que se decida cambiar el voto $y$, posteriormente, se racionalice esa decisión.

No es posible saber de un modo definitivo qué refleja la variable LRPP21. Para ello sería necesario una observación más, previa a las elecciones, para determinar si el cambio de ubicación en la escala ideológica precedió al voto o, al contrario, sucedió, al cambio de voto ${ }^{37}$. Con todo, utilizando la evidencia estadística y lógica disponible es posible ofrecer una interpretación.

En primer lugar, la probabilidad de votar finalmente al PSOE no está asociada de un modo positivo solamente con el hecho de situar al PP más a la derecha. La ubicación de IU más a la izquierda hace también más probable que el votante cambie su voto. La campaña del PSOE se centró en marcar la identidad de derechas del PP y no se refirió a la naturaleza de la coalición IU. Entonces, si situar al PP más a la derecha y a IU fueron consecuencia del mismo mecanismo, éste no pudo ser la retórica desarrollada durante la campaña electoral ${ }^{38}$.

En segundo lugar, las interacciones de las variables LRIU21 y LRPP21 con CAMINT no son significativas (Modelo 2). Se podría pensar que es así porque estas interacciones son colineales con las variables sin interacciones. Por ello he estimado un modelo unicamente con las interacciones (Modelo 3). Como se puede observar en la Tabla 1, estas interacciones no son significativas. Es decir, la influencia de extremar la posición en la escala ideológica de estos partidos sobre la probabilidad de cambio de voto no fue diferente para los votantes influidos por la campaña electoral que para los inmunes a la misma.

Finalmente, la decisión de los votantes reticentes, se acomoda a las consecuencias de la eliminación de disonancia cognitiva caracterizadas en algunos modelos deductivos (vid. Akerloff y Dickens, 1982; Rabin, 1994). Recordemos las consideraciones acerca de la eliminación de la disonancia cognitiva, en concreto la expresión (3). Partiendo del supuesto de que las creencias de los individuos no están totalmente determinadas, sino que éstos tienen cierto control sobre las mismas, en esta expresión se observa cómo el cambio de creencias puede permitir al individuo eliminar la disonancia de una acción y determinar que la utilidad neta que obtiene de la misma sea mayor que la que tenía antes de tal cambio. La decisión de los votantes reticentes fue el resultado de una enorme tensión entre la evaluación de la actuación gubernamental y la necesidad de tener que votar al "mejor tipo". Su decisión final de votar al PSOE no fue simplemente una opción

\footnotetext{
${ }^{37}$ Para ser más precisos, sería necesaria una observación más, previa al momento en que el individuo toma su decisión (final) de voto.

${ }^{38}$ Obviamente, podría haber ocurrido que las causas de la polarización de PP e IU hubieran sido diferentes.
} 
RIS

por la coherencia con sus características y disposiciones políticas Barreiro y Sánchez-Cuenca, 1998) como consecuencia de la activación de la campaña electoral (Fernández, 2001). Si finalmente decidieron votar al PSOE fue por 1) el cambio del sentido del voto de retrospectivo a prospectivo, y 2) porque el equilibrio fue favorable al PSOE. Ahora bien, que la evaluación retrospectiva de la economía no influyera en su decisión final de voto no implica que olvidaran su evaluación retrospectiva negativa de la actuación gubernamental. Tampoco implica que la campaña mejorara esta evaluación ${ }^{39}$. En efecto, como señalan Arango y Díez (1993: 15), estos votantes "se veían confrontados a una búsqueda subjetiva del sentido de su voto para que éste no fuera disonante con su valoración de la situación (económica y política) ni con sus ideas politicas". En esta situación es muy probable que estos votantes racionalizaran su opción final. Y qué mejor que hacerlo a través de una variable determinante del comportamiento electoral en España: la ideología (Gunther y Montero, 1993; Lancaster y Lewis-Beck, 1986; Sani y Montero, 1986).

Ahora bien, aun no siendo la variable que recoge la polarización ideológica del PP un efecto de la retórica, esto no significa que la inclusión de esta dimensión en la campaña no influyera en la decisión final de los votantes cambiantes. Su efecto pudo acomodarse a mecanismos propios de maniobras herestéticas. Una vez conseguido que el voto fuera prospectivo, el equilibrio fue favorable al PSOE gracias a: a) incidir en la falta de credibilidad del PP, y b) a la introducción de la dimensión izquierda-derecha en la competición.

\section{CONCLUSIONES}

En este trabajo se ha presentado un análisis detallado de un caso. Se ha mostrado cómo un partido evita ser accountable ante los ciudadanos. Explotando la existencia de un partido aspirante lejano a un grupo de votantes, consigue cambiar el sentido del voto de estos ciudadanos - que los criterios prospectivos sustituyan a los retrospectivos en la formación su decisión-y desarrolla maniobras herestéticas consistentes en resaltar la dimensión ganadora, la dimensión por la que estos ciudadanos están tan alejados del partido aspirante. De este modo, el partido en el gobierno indujo un equilibrio que le fue favorable.

Este resultado es simplemente una ilustración de cómo los partidos pueden escapar al control de los ciudadanos. Aporta, además, una clara muestra de las

\footnotetext{
${ }^{39}$ En efecto, de acuerdo nuevamente con el modelo econométrico, la interacción del efecto de la campaña con la evaluación de la actuación gubernamental no está asociada de un modo significativo con el cambio de voto (Modelo 5).
} 
dificultades de concebir las elecciones como una institución que produce la responsabilidad de los gobiernos ante los ciudadanos. Y, si bien las conclusiones del análisis son contingentes a unas elecciones concretas, tienen implicaciones para conocer las consecuencias en términos de accountability de la existencia de un partido aspirante que es considerado un "mal tipo" por el electorado o por un conjunto del mismo. En efecto, podríamos partir de una especificación estratégica de la decisión del votante. Ésta consistiría en la especificación de las utilidades esperadas que obtiene el individuo cuando vota en un sentido retrospectivo y cuando lo hace prospectivamente. Partiendo de esta especificación, habría que realizar análisis de estática comparativa para determinar la influencia sobre el sentido del voto de la combinación de una mala evaluación retrospectiva de la actuación del partido en el gobierno y la existencia de un partido aspirante considerado un "mal tipo". Pero esto excede el propósito de este trabajo.

\section{REFERENCIAS BIBLIOGRÁFICAS}

AKERLOFF, G. y W. DICKENS (1982), "The Economic Consequences of Cognitive Dissonance", American Economic Review, vol. 72.3, pp. 307-319.

ALDRICH, J. (1997), "When is it rational to vote?", en D. Mueller (ed.), Perspectives on Public Choice: $A$ Handbook, Cambridge, Cambridge University Press.

ALESINA, A. y H. ROSENTHAL (1995), Partisan Politics, Divided Governments, and the Economy, Cambridge, Cambridge University Press.

ARANGO, J. y M. DÍEZ (1993), “6-J: El sentido de una elección”, Claves de Razón Práctica, vol. 36 , pp. 10-18.

BARREIRO, B. e I. SÁNCHEZ-CUENCA(1998), “Análisis del cambio de voto hacia el PSOE en las elecciones de 1993”, Revista Española de Investigaciones Sociológicas, vol. 82, pp. 191-211.

DOWNS, A. (1957), An Economic Theory of Democracy, Nueva York, Harper and Row.

FEARON, J. (1999), "Electoral Accountability and the Control of Politicians: Selecting Good Types versus Sanctioning Poor Performance", en Przeworski, A., S. Stokes and B. Manin (eds.), Democracy, Accountability and Representation, Cambridge, Cambridge University Press.

FEREJOHN, J. (1986), "Incumbent Performance and Electoral Control”, Public Choice, vol. 50, pp. $5-25$.

FERNÁNDEZ, M. (2001), “¿Para qué sirven las campañas electorales? Los efectos de la campaña electoral española de 1993", Revista Española de Investigaciones Sociológicas, vol. 93: 61-87.

FINKEL, S. (1993), "Campaign Effects on Voter Choice in the German Election of 1990", The Journal of Politics, vol. 55.1, pp. 1-21. 
RIS

REVISTA INTERNACIONAL DE SOCIOLOGIA

№ 41, MAYO-AGOST0, 2005

JUAN RAFAEL MORILLAS MARTINEL

FINKEL, S. y P. SCHROTT (1995), "Campaign Effects on Voter Choice in the German Election of 1990”, British Journal of Political Science, vol. 25.3, pp. 349-378.

FIORINA, M. (1981), Retrospective Voting in American National Elections, New Haven, Yale University Press.

GUNTHER, R. y J.R. MONTERO (1993), "Los anclajes del partidismo. Un análisis comparado del comportamiento electoral en cuatro democracias del sur de Europa", en P. del Castillo (ed.), Comportamiento politico y electoral, Madrid, CIS.

KEY, V.O. (1966), The Responsible Electorate, Nueva York, Vintage Books.

LANCASTER, TH. Y M. LEWIS-BECK (1986), "The Spanish Voter: Tradition, Economics and Ideology", The Journal of Politics, vol. 48, pp. 648-674.

LAZARSFELD, P. et al., (1968), The People Choice, Nueva York, Columbia University Press.

MANIN, B., A. PRZEWORSKI y S. STOKES (1999), “Elections and Representation”, en Przeworski, A., S. Stokes and B. Manin (eds.), Democracy, Accountability and Representation, Cambridge, Cambridge University Press.

MARAVALL, J.M. (1999), “Accountability and Manipulation", en Przeworski, A., S. Stokes and B. Manin (eds.), Democracy, Accountability and Representation, Cambridge, Cambridge University Press.

(2003), El control de los politicos, Madrid, Alianza.

MARÍ-KLOSE, P. (1997), "Elecciones críticas, votantes críticos, campañas críticas. Consecuencias electorales de la campaña del PSOE en 1993", manuscrito.

MARKUS, G. y P. CONVERSE (1979), "A Dynamic Simultaneous Equation Model of Electoral Choice", American Political Science Review, vol. 73, pp. 1055-1070.

RABIN, M. (1984), "Cognitive dissonance and social change", Journal of Economic Behaviour and Organization, vol. 23.2, pp. 177-194.

RIKER, W. (1982), Liberalism Against Populism. A Confrontation of the Theory of Democracy and the Theory of Social Choice, San Francisco, W.H. Freeman.

(1986), The Art of Political of Political Manipulation, New Haven, Yale University Press.

(1990), "Heresthetic and Rethoric in the Spatial Model", en J. Enelow y M. Hinich (eds.), Advances in the Spatial Theory of Voting, Cambridge, CUP.

SANI, G. y J.R. MONTERO (1986), "El espectro político: izquierda, derecha y centro", en J.J. Linz y J.R. Montero (eds.), Crisis y cambio. Electores y Partidos en la España de los años ochenta, Madrid, CEC. 